Letter to the Editor

\title{
A case of lignocaine toxicity
}

Navin Kumar Devaraj

Department of Family Medicine, Faculty of Medicine and Health Sciences, University Putra Malaysia, Serdang, Selangor Darul Ehsan, Malaysia

Submitted: 4 February 2017

Accepted: 7 March 2017

Arch Med Sci Civil Dis 2017; 2: e48-e49

DOI: https://doi.org/10.5114/amscd.2017.66629

Copyright (c) 2017 Termedia \& Banach

Cases of local anesthesia toxicity are rare and infrequently reported. But what the author experienced in this case was a real eye opener and a great teaching experience which can be applied to future similar presentations.

A 27-year-old Pakistani man presented to the primary care clinic complaining of accidentally injuring his right forearm while repairing his house roof. Part of the middle third of his right forearm was cut by a sharp piece of zinc used for roof repair. Initially he had some bleeding which subsided by self bandaging of the wound.

Vital signs were stable. Examination of his right forearm showed a vertical laceration wound $4 \times 1 \mathrm{~cm}$ over the middle third of the dorsal aspect. The cut was deep, reaching to the tendon sheath of one of the tendons, suspected to be the extensor digitorum muscle tendon. However, the tendon appeared intact. Fingers and wrist range of motion were full. Sensation over the right hand was intact and perfusion was good. Both radial and ulnar pulses were felt and were normal.

After obtaining informed consent from the patient, $5 \mathrm{ml}$ of local lidocaine $2 \%$ injection was given over the laceration wound. To enhance the anesthetic effect, topical lignocaine of the same concentration was sprayed over the injury site. Toileting and suturing (T\&S) was then done with a total of 4 deep catgut continuous stitches and 4 superficial nylon sutures. After surgery, an abnormal finding was noted. The patient was unable to extend his right ring finger at the metacarpophalangeal joint. Sensation was still intact. There was fear was that maybe a tendon cut was missed. However, miraculously, after $15 \mathrm{~min}$, the patient regained the extension function of his right ring finger. The patient was discharged with analgesics and antibiotics. He was told to return after 2 days for wound inspection. The patient was advised to return immediately should the above problem recur. On examination 2 days later, the finger's extension remained intact and, needless to say, the removal of stitches was also uneventful.

What was supposed to be a routine T\&S turned out to be a minor scare. This was the first time in the author's experience that temporary palsy of a finger or toes appeared after injection or spray of a local anesthetic. The author does not usually use topical local anesthetic spray as a local anesthesia but decided to use it this time to enhance the anesthesia over the injury site. This shows that even topical anesthetic spray has a powerful numbing and absorptive effect. Clearly the spray had inadvertently and excessively numbed the radial nerve and therefore produced temporary paralysis of the right ring finger's extensor digitorum muscle.

Going back to the dreaded anatomy, this muscle is the main extensor of the fingers. Its proximal attachment is at the lateral epicondyle and
Corresponding author:

Dr. Navin Kumar Devaraj Department of Family Medicine

Faculty of Medicine and Health Sciences University Putra Malaysia 43400 Serdang

Selangor Darul Ehsan, Malaysia

Phone: +6013-3105381

E-mail: knavin@upm.edu.my 
the distal attachment is at the extensor hoods of the fingers. It is innervated by the deep branch of the radial nerve [1].

Each pump spray of topical lignocaine delivers $10 \mathrm{mg}$ per dose [2]. Compare that with $5 \mathrm{ml}$ of subcutaneous lignocaine that has already delivered $100 \mathrm{mg}$, which is $1 / 3^{\text {rd }}$ of the recommended manufacturer's maximum dose of $300 \mathrm{mg}$. Certainly a topical spray, although less invasive, increases the chance of toxicity. The risk of toxicity is increased by the amount and route of administration of local anesthesia used and the notable absence of a vasoconstrictor such as adrenaline [3].

In conclusion, the learning point in this case is that although local anesthetic injection use is common and relatively safe, it should be treated as a drug that has potential to cause toxicity at high levels. The use of topical lignocaine injection as an adjunct in this case was most inappropriate as the patient had already been given subcutaneous injection of a similar drug.

\section{Acknowledgments}

The author would like to thank the patient for his permission to publish his case and for providing the author with a great teaching experience.

\section{Conflict of interest}

The authors declare no conflict of interest.

\section{References}

1. Muscles in the Posterior Compartment of the Forearm. Available at: teachmeanatomy.info. Accessed: 3 January 2017.

2. Plessis RD. Local anaesthetics: characteristics, uses and toxicities. CME 2009; 27: 398-400.

3. Mehra P, Caiazzo A, Maloney P. Lidocaine toxicity. Anesth Prog 1998; 45: 38-41. 